\title{
State of Charge Estimation Framework for Lithium-ion Batteries Based on Square Root Cubature Kalman Filter under Wide Operation Temperature Range
}

\author{
Jiangwei Shen ${ }^{1}$, Jian Xiong ${ }^{1}$, Xing Shu ${ }^{1}$, Guang $\mathrm{Li}^{2}$, Yuanjian Zhang ${ }^{3}$, Zheng Chen ${ }^{1,2^{*}}$ and Yonggang Liu ${ }^{4 * *}$ \\ ${ }^{1}$ Faculty of Transportation Engineering, Kunming University of Science and Technology, Kunming 650500, China \\ ${ }^{2}$ School of Engineering and Materials Science, Queen Mary University of London, London, E1 4NS, United \\ Kingdom \\ ${ }^{3}$ School of Mechanical and Aerospace Engineering, Queen's University Belfast, BT9 5AG, Northern Ireland \\ ${ }^{4}$ State Key Laboratory of Mechanical Transmissions \& School of Automotive Engineering, Chongqing University, \\ Chongqing, 400044, China \\ Email: shenjiangwei6@163.com, xjebox@qq.com, shuxing92@kust.edu.cn, g.li@qmul.ac.uk, chen@kust.edu.cn, \\ andylyg@umich.edu \\ Corresponding Author: Zheng Chen (chen@kust.edu.cn), and Yonggang Liu (andylyg@umich.edu)
}

\begin{abstract}
Due to the significant influence of temperature on battery charging and discharging performance, exact evaluation of state of charge under complex temperature environment becomes increasingly important. This paper develops an advanced framework to estimate the state of charge for lithium-ion batteries with consideration of temperature variation. Firstly, an accurate electrical model with wide temperature compensation is established, and a series of experiments are carried out under wide range time-varying temperature from $-20{ }^{\circ} \mathrm{C}$ to $60{ }^{\circ} \mathrm{C}$. Then, the genetic algorithm is leveraged to identify the temperaturedependent model parameters. On this basis, the battery state of charge is accurately estimated based on the square root cubature Kalman filter algorithm. Finally, the availability of the proposed method at different temperatures is validated through a complicated mixed working cycle test, and the experimental results manifest that the devised framework can accurately evaluate state of charge under wide time-varying temperature range with the maximum error of less than $2 \%$.
\end{abstract}

Key words: state of charge, temperature compensation model, time-varying temperature, lithium-ion battery

\section{INTRODUCTION}

Owing to the merits of long service lifetime and high energy density, lithium-ion (Li-ion) batteries have been progressively considered as the mainstream energy storage solutions, especially in electric vehicles (EVs) applications [1]. However, improper utilization of Li-ion batteries may result in thermal runaway, fire or explosion, significantly affecting operation safety of EVs. To pledge safe and efficient operation of Li-ion batteries, increasingly high requirements of monitoring internal state and ensuring safe operation have been imposed on battery management system (BMS) [2]. As a critical task of BMS, accurate estimation of state of charge (SOC) can supply the information of how much electric capacity is left in the battery and how long the 
battery can be fully charged [3]. In addition, accurate SOC knowledge can be conducive to avoidance of abuse operation, such as over-charge/discharge, and supply valuable reference for state of health (SOH) prediction and cell balance management in a battery pack. Usually, SOC can be expressed by the ratio of the remaining available capacity over the nominal capacity [4]. Obviously, SOC cannot be measured directly but only be estimated indirectly from the measured variables such as voltage, current and temperature [5]. Moreover, SOC can be significantly affected by external operating conditions (e.g. time-varying load current and ambient temperature), leading to certain difficulty of robust estimation [6]. How to accurately estimate the SOC under time-varying conditions becomes one of the main bottlenecks in BMS [7].

In recent years, many researchers have paid much attention to battery SOC estimation, and a mass of advanced methods have been promoted, mainly including coulomb counting method, characteristic parameter methods, machine learning-based methods and filtering algorithm-based methods [8]. In [9], a comprehensive investigation of the most commonly used battery modeling and state estimation methods for BMSs is performed. The coulomb counting method has been widely employed in practice due to its simplicity and easy implementation. However, its accuracy heavily depends on the fidelity of measured parameters and initial SOC value [10]. The commonly used characteristic parameter-based methods contain internal resistance method, chemical impedance spectroscopy method and open circuit voltage (OCV) method. However, these methods cannot estimate SOC online due to the limitation of test conditions and cannot be applied in EVs directly [11] Machine learning-based methods can reveal the implicit relationship between characteristic parameters and SOC [12]. It does not require the prior knowledge of internal chemical or physical characteristics of Li-ion batteries and therefore features an acceptable estimation accuracy [8]. Nonetheless, the machine learning-based methods require sufficient offline data for model training to reveal the underlying dynamic characteristics of $\mathrm{Li}$ ion batteries. Filtering algorithm-based methods in combination with different battery models, has become the research hotspot and features high estimation accuracy of SOC, owing to its preferable adaption capabilities and strong robustness [13]. However, these approaches can be influenced due to the model parameter variation, which are significantly affected by ambient temperature, aging state [14] and loading profiles [15]. The commonly used filter algorithms include Kalman filter (KF) family [16], sliding mode observer (SMO) [17] and particle filter (PF) [18]. In addition, the Luenberger observer and least square-based method are also adopted in SOC estimation [19]. Amongst them, KF and its extension have been widely investigated and employed. Ref. [20] investigates the estimation performance of unscented KF (UKF) and extended KF (EKF) for SOC on the 
basis of constructing a Thevenin equivalent circuit model (ECM), and the experimental results indicate that the UKF algorithm leads to better precision and faster correction speed than the EKF algorithm. In [21], to cope with the variation of noise covariance matrices, an adaptive covariance calculation based EKF (AEKF) is introduced to estimate the SOC of Li-ion batteries. The results indicate that the AEKF algorithm raises better noise suppression, and the evaluating error is less than $2 \%$. In [22], the cubature $\mathrm{KF}$ (CKF), derived from the radial volume criterion of the third-order sphere, is employed to estimate SOC. It can utilize a group of volume points to border on the error covariance of nonlinear systems under the existence of additional Gaussian noise, and is quirt close to Bayesian filtering to conduct state estimation of nonlinear systems [23]. For the sake of reducing the computational burden and enhancing the stability of CKF, a square root CKF (SRCKF) algorithm is proposed in [24], which updates error covariance matrix based on the square root filtering technique, thereby solving the non-positive definite and non-asymmetric problems of error covariance matrix caused by errors rounding and improving the real-time performance of CKF.

Although massive attempts have been made in SOC estimation under certain conditions, there still exists a gap to be filled when considering the temperature variation, especially under the condition of wide timevarying ranges. Due to the instable working conditions of EVs, the battery temperature changes dramatically within a large range, leading to certain difficulty when conducting robust SOC estimation [25]. By considering the temperature influence, Ref. [26] establishes a temperature compensation model, considering the range of 37 ${ }^{\circ} \mathrm{C}$ to $40{ }^{\circ} \mathrm{C}$ with an interval of $1{ }^{\circ} \mathrm{C}$, to estimate $\mathrm{SOC}$ via the EKF. In [27], to promote the SOC estimation precision at different temperatures, temperature-varying parameters of the built electrical model are updated dynamically by introducing a compensation factor. In [28], a temperature compensation model mainly accounting for the temperature variation of $0{ }^{\circ} \mathrm{C}$ to $40{ }^{\circ} \mathrm{C}$ is built based on a two-order ECM, and then the SOC is estimated by the UKF, which declares that the maximum estimation error is less than $3 \%$. In practical applications, the battery temperature dynamically changes within a large range (usually between $10{ }^{\circ} \mathrm{C}$ and 50 ${ }^{\circ} \mathrm{C}$ ), thus significantly affecting estimation accuracy of SOC [29]. Thus, it is imperative to establish a temperature-dependent electrical model to cover the whole operation temperature range [30], and then the SOC can be estimated robustly with the help of advanced filters [31].

Motivated by this, an electrical model with wide temperature compensation is established on the basis of a two-order resistance capacitance (RC) ECM, for fully accounting for the evolution of ambient temperature and resolving the low efficiency of battery model under wide temperature ranges. Moreover, the battery capacity, 
OCV are tested under the wide temperature range from $-20{ }^{\circ} \mathrm{C}$ to $60{ }^{\circ} \mathrm{C}$ with $10{ }^{\circ} \mathrm{C}$ as an interval, and the accurate model parameters are obtained through genetic algorithm (GA). Then, the SRCKF method with the fusion of temperature variation is advanced to estimate the battery SOC. The experimental validations are carried out at different temperatures and compared with the commonly addressed methods to emphasize the advancement of the presented framework.

The residual of this paper is listed hereafter: the ECM, battery experiment and parameters identification are elaborated in Section II. The flowchart of SRCKF algorithm and estimation steps of SOC are detailed in Section III. In Section IV, the established model and the proposed SOC estimation method are verified and discussed under complex environmental temperatures. Section V draws the main conclusions of this paper.

\section{BATTERY MODELING AND CHARACTERIZATION TEST}

\section{A. Battery Modeling}

The battery models can be generally summarized into three categories: ECM, electrochemical model and data driven model [32]. Although the electrochemical model and data-driven model exhibit high accuracy in the whole temperature range, they are often limited in practical applications due to the heavy calculation intensity and massive experimental data requirements. However, the ECM is symbolized by electronic components to simulate the electrical behaviors of Li-ion batteries, and can easily and simply convert the internal chemical reaction into intuitive external characteristics, thus being widely adopted in practice [33]. In particular, twoorder RC ECM can clearly pretend the concentration difference polarization and electrochemical polarization characteristics of Li-ion batteries to improve the model accuracy without much increase of complexity [34]. As such, it is employed in this study for modeling the battery and consequently facilitating the SOC estimation, as shown in Fig. 1.

In Fig. $1, U_{t}(t)$ means the terminal voltage, $I(t)$ denotes the current, $U_{1}(t)$ and $U_{2}(t)$ are the voltage drop of the corresponding RC network respectively, $R_{0}$ represents the ohmic internal resistance, $R_{j}$ and $C_{j}(j=1,2)$ denote the polarization resistance and capacitance of the battery, respectively, $U_{O C}(t)$ denote the $\mathrm{OCV}$, which is a nonlinear function of battery SOC and temperature $T$, as:

$$
U_{O C}(t)=f_{o c v}(S O C, T)
$$

According to the Kirchhoff law, the following state equations can be yielded, as: 


$$
\left\{\begin{array}{l}
\frac{d U_{1}}{d t}=\frac{I(t)}{C_{1}}-\frac{U_{1}}{R_{1} C_{1}} \\
\frac{d U_{2}}{d t}=\frac{I(t)}{C_{2}}-\frac{U_{2}}{R_{2} C_{2}} \\
U_{t}=U_{O C}(t)-I(t) R_{0}-U_{1}-U_{2}
\end{array}\right.
$$

Based on the Coulomb counting, the SOC can be obtained as:

$$
\operatorname{SOC}(k+1)=\operatorname{SOC}(k)+\frac{\Delta t \eta}{C_{n}} \int I(k) d t
$$

where $C_{n}$ denotes the battery nominal capacity, $\Delta t$ and $\eta$ represent the sampling interval and coulomb efficiency, respectively.

\section{B. Experiments and Parameters Identification}

In this study, a lithium nickel cobalt manganese (NCM) oxide battery with the nominal capacity of $4 \mathrm{Ah}$ is selected as the experimental object, and its main specifications are displayed in Table I. To investigate the charging and discharging characteristics of batteries in a wide temperature range, the experimental tests are carried out from $-20{ }^{\circ} \mathrm{C}$ to $60{ }^{\circ} \mathrm{C}$ with an interval of $10{ }^{\circ} \mathrm{C}$, and include the capacity test, hybrid pulse power characterization (HPPC) experiments and different dynamic current cycles tests. Note that the sampling frequency is set to $1 \mathrm{~Hz}$ during the battery test.

\section{1) Capacity Test}

The capacity test is conducted with a temperature increase step of $10{ }^{\circ} \mathrm{C}$ from $-20^{\circ} \mathrm{C}$ to $60{ }^{\circ} \mathrm{C}$, and the test procedures are detailed as below: 1) Charge the battery with the constant-current and constant-voltage (CC-CV) charge mode. Note that the constant current charge rate is $2 \mathrm{~A}$, the cut-off voltage and current in this step are 4.2 V and 0.08 A, respectively. 2) Shelve the battery for one-hour rest. 3) Discharge the battery with 0.8 A current until the voltage declines to 2.75 V. 4) Repeat steps 1) to 3) for three times. Then, the maximum available capacity can be obtained by averaging the three experimental results. The results of discharge capacity test are exhibited in Fig. 2 (a). As can be observed, the available discharge capacity is less at both high and low temperature conditions. When the battery works within $30^{\circ} \mathrm{C}$ to $50{ }^{\circ} \mathrm{C}$, the discharge capacity is almost the same as the nominal capacity. However, the discharge capacity decreases by $46.08 \%$ rapidly at $-20{ }^{\circ} \mathrm{C}$, compared with the nominal capacity. The discharging voltage curves at $-20{ }^{\circ} \mathrm{C}$ to $60{ }^{\circ} \mathrm{C}$ are depicted in Fig. 2 (b), in which we can find that the voltage drops with a fast speed at lower temperature, resulting in less discharge capacities. This phenomenon can be explained by the increase of internal resistance at low temperature. Moreover, the 
experimental results indicate that the proper operating temperature of the battery is between $30{ }^{\circ} \mathrm{C}$ and $50{ }^{\circ} \mathrm{C}$.

\section{2) OCV Test}

In this study, according to the test method in [35], the OCV test is conducted at different temperatures for estimating the SOC. Fig. 3 sketches the three-dimensional nonlinear relationship between OCV, SOC and temperature. As can be found, the relationship curves between OCV and SOC are almost consistent with the variation of temperature. To intuitively describe the functional relation between OCV and SOC, a mathematical polynomial equation can be exploited to fit the experimental data, as shown in (4). From Fig. 3, we can find that when the battery SOC is higher than $60 \%$, the OCV increases gradually with the increase of temperature, whereas the OCV shows decrease trend with the raise of temperature under the low SOC. This indicates that the OCV can be affected by temperature in different SOC stages.

$$
f_{\text {OCV }}(\mathrm{s})=p_{1} s^{5}+p_{2} s^{4}+p_{3} s^{3}+p_{4} s^{2}+p_{5} s^{1}+p_{6}
$$

\section{3) HPPC Test}

HPPC test can activate the polarization of Li-ion inside the battery through large pulse charging and discharging profiles. By means of the HPPC experimental test and corresponding identification algorithm, the model parameters can be easily identified [36]. In this study, the HPPC test are carried out under different temperatures, and the related results at $25^{\circ} \mathrm{C}$ is shown in Fig. 4 (a). In this study, GA is leveraged to acquire the

parameters of ECM, which contains $R_{0}, R_{1}, R_{2}, C_{1}$ and $C_{2}$ in (2). GA is a kind of artificial intelligence method which simulates the biological evolution to achieve the global optimization. The GA algorithm shows predominant performance in both local and global parameters estimation of nonlinear systems. Although the GA algorithm requires high operation time and is difficult to attain online application, it can still be employed for model parameter identification due to its strong identification capability [37]. The identification consequences are demonstrated in Fig. 4 (b) to (f). It can be found that with the rise of temperature, $R_{0}, R_{1}$ and $R_{2}$ decrease rapidly; while the polarization capacitance $C_{1}$ and $C_{2}$ increase slowly. The parameters variation trend shown in Fig. 4 indicates that the battery's charge/discharge property and model parameters are dramatically influenced by temperature variation.

In the next step, the process of applying the SRCKF to estimate SOC will be detailed, and the battery model parameters will be dynamically updated according to the present temperature. 


\section{SOC ESTIMATION ALGORITHM}

Compared with the conventional EKF and its family algorithms, SRCKF can not only solve the problem of inferior accuracy of Taylor expansions for EKF, but also overcome the non-positive definite and nonasymmetric problems of error covariance matrix caused by error rounding for UKF and CKF. Although the PF algorithm demonstrates high SOC estimation accuracy, it needs a large number of samples, and the calculation labor is still burdensome. By considering these merits, the SRCKF algorithm is exploited for the SOC estimation in this study. The flowchart of the developed SOC estimation framework is sketched in Fig. 5.

For a nonlinear system, the discretized state equation and output equation are formulated as:

$$
\left\{\begin{array}{l}
X_{k+1}=A_{k} X_{k}+B_{k} u_{k}+\omega_{k} \\
Y_{k}=C_{k} X_{k}+D_{k} u_{k}+v_{k}
\end{array}\right.
$$

where $u_{k}$ and $Y_{k}$ are the input and output, respectively, $X_{k}$ means the state vector at step $k . \omega_{k}$ and $v_{k}$ represent the system and measurement noise, and their covariance are $Q_{k}$ and $R_{k}$, respectively. $A_{k}, B_{k}$, $C_{k}$ and $D_{k}$ individually express the transfer, control, observation and feedforward matrixes of the system, as:

$$
\begin{gathered}
Y_{k}=f\left(S O C_{k}\right)-U_{1, k}-U_{2, k}-R_{0} I_{k} \\
u_{k}=I_{k} \\
A_{k}=\left[\begin{array}{ccc}
s_{1} & 0 & 0 \\
0 & s_{2} & 0 \\
0 & 0 & 1
\end{array}\right] \\
B_{k}=\left[\begin{array}{ccc}
R_{1}\left(1-S_{1}\right) & R_{2}\left(1-S_{2}\right) & 1 / C_{n}
\end{array}\right]^{T} \\
C_{k}=\left[\begin{array}{ccc}
1 & 1 & \left.\frac{\partial f_{o c v}(S O C)}{\partial S O C}\right|_{s o c=s o c_{k}}
\end{array}\right] \\
D_{k}=R_{0, k}
\end{gathered}
$$

where $s_{1}=\exp \left(-1 / R_{1} C_{1}\right)$, and $s_{2}=\exp \left(-1 / R_{2} C_{2}\right)$. The implemention procedure of SOC estimation based on the SRCKF algorithm can be summarized as below:

Step 1. Initialization.

$$
\hat{x}_{0}=E\left[X_{0}\right], P_{0}=E\left[\left(X_{0}-\hat{X}_{0}\right)\left(X_{0}-\hat{X}_{0}\right)^{T}\right]
$$

where $E(\cdot)$ represents the expectation, and $P_{0}$ means error covariance matrix. 
Step 2. Generation of basic volume points $\xi_{i}$.

$$
\xi_{i}=\sqrt{\frac{m}{2}}[1]_{i}
$$

where [1] denotes the complete and fully symmetric point set, which is the point set generated by arranging the elements of $\mathrm{n}$-dimensional unit column vector and changing the element symbols.

Step 3. Time domain update.

Step 3. 1. Calculate the sample points, as:

$$
X_{i, k-1 \mid k-1}=S_{k-1 \mid k-1} \xi_{i}+\hat{x}_{k-1 \mid k-1}
$$

where $\hat{x}_{k-1 \mid k-1}$ and $S_{k-1 \mid k-1}$ represent the square root form of state error covariance and the predicted state at step $k-1$, respectively.

Step 3. 2. Estimate state at each sampling point, as:

$$
X_{i, k \mid k-1}^{*}=f\left(X_{i, k-1 \mid k-1}, u_{k}\right)
$$

Step 3. 3. Calculate the next predicted value, as:

$$
\hat{x}_{k \mid k-1}=\frac{1}{m} \sum_{i=1}^{m} X_{i, k \mid k-1}^{*}
$$

Step 3. 4. The square root of covariance matrix for state estimation error can be calculated, as:

$$
\begin{gathered}
X_{k \mid k-1}^{*}=\frac{1}{\sqrt{m}}\left[X_{1, k \mid k-1}^{*}-\hat{x}_{k \mid k-1}, \cdots, X_{m, k \mid k-1}^{*}-\hat{x}_{k \mid k-1}\right] \\
S_{k \mid k-1}=\operatorname{tria}\left(\left[X_{k \mid k-1}^{*}, S_{Q, k-1}\right]\right)
\end{gathered}
$$

where tria represents the QR decomposition, and $S_{Q, k-1}$ is the square root form of process noise $Q_{k-1}$. Step 4. Measurement update.

Step 4. 1. Calculate the input sampling points of the measurement equation, as:

$$
X_{i, k \mid k-1}=S_{k \mid k-1} \xi_{i}+\hat{x}_{k \mid k-1}
$$

Step 4. 2. Estimate the measurement output at each sampling point, as:

$$
Z_{i, k \mid k-1}=h\left(X_{i, k \mid k-1}, u_{k}\right)
$$

Step 4. 3. Calculate the predicted value of the observed output, as: 


$$
\hat{z}_{k \mid k-1}=\frac{1}{m} \sum_{i=1}^{m} Z_{i, k \mid k-1}
$$

Step 4. 4. Calculate the square root of the observation error covariance $S_{z z, k \mid k-1}$, as:

$$
\left\{\begin{array}{l}
S_{z z, k \mid k-1}=\operatorname{tria}\left(\left[\xi_{k \mid k-1}, S_{R, k}\right]\right) \\
\xi_{k \mid k-1}=\frac{1}{\sqrt{m}}\left[Z_{1, k \mid k-1}-\hat{z}_{k \mid k-1}, \cdots, Z_{m, k \mid k-1}-\hat{z}_{k \mid k-1}\right]
\end{array}\right.
$$

where $S_{R, k}$ is the square root of measurement noise $R_{k}$.

Step 4. 5. Calculate the covariance of state and observation output error $P_{x z, k \mid k-1}$, as:

$$
\begin{gathered}
\chi_{k \mid k-1}=\frac{1}{\sqrt{m}}\left[X_{1, k \mid k-1}-\hat{x}_{k \mid k-1}, \cdots, X_{m, k \mid k-1}-\hat{x}_{k \mid k-1}\right] \\
P_{x z, k \mid k-1}=\chi_{k \mid k-1} \xi_{k \mid k-1}^{T}
\end{gathered}
$$

Step 4. 6. Calculate the filter gain $W_{k}$, as:

$$
W_{k}=\frac{P_{x z, k \mid k-1} / S_{z z, k \mid k-1}^{T}}{S_{z z, k \mid k-1}}
$$

Step 4. 7. Update the status, as:

$$
\hat{x}_{k \mid k}=\hat{x}_{k \mid k-1}+W_{k}\left(z_{k}-\hat{z}_{k \mid k-1}\right)
$$

Step 4. 8. Update the square root form of state error covariance, as:

$$
S_{k \mid k}=\operatorname{tria}\left(\left[\chi_{k \mid k-1}-W_{k} \xi_{k \mid k-1}, W_{k} S_{R, k}\right]\right)
$$

In the next step, the model will be firstly validated, and the model parameters will be analyzed, followed by the substantial SOC estimation validation.

\section{EXPERIMENTAL VALIDATION AND DISCUSSION}

In this section, the constructed battery model is firstly validated, and then the proposed method is compared with the other conventional SOC estimation methods, followed by the SOC evaluation at different ambient temperatures.

\section{A. Model Validation}

To prove the validity of the constructed model, the battery is fully charged, followed by the urban dynamometer driving schedule (UDDS) test. The results at $20^{\circ} \mathrm{C}$ are shown in Fig. 6 . Note that the voltage error 
in this paper is defined as that in (28). Fig. 6 (a) shows that the estimated voltage can track the measurement value at $20^{\circ} \mathrm{C}$ with high accuracy, even when the battery current fluctuates quickly. Fig. 6 (b) demonstrates that the maximum relative error at $-10{ }^{\circ} \mathrm{C}, 0{ }^{\circ} \mathrm{C}, 20{ }^{\circ} \mathrm{C}, 40{ }^{\circ} \mathrm{C}$ and $60{ }^{\circ} \mathrm{C}$ is $1.70 \%, 1.21 \%, 0.60 \%, 1.14 \%$ and $1.59 \%$, respectively. The results manifest that the constructed ECM integrating temperature compensation can faithfully simulate the dynamic and static characteristics of Li-ion batteries in a wide temperature range.

$$
\text { error }_{t}=\frac{U_{\text {simulation }, t}-U_{\text {measuerd }, t}}{U_{\text {measuerd }, t}} \times 100 \%
$$

\section{B. The Comparison of Different Methods}

To authenticate the predominance of presented SOC estimation framework, another two filters, namely EKF and AEKF are implemented to predict SOC, and their estimation results are evaluated and compared with that of the SRCKF algorithm. The ambient temperature is set to $25^{\circ} \mathrm{C}$ during the test, and the current data obtained by UDDS are repeatedly imposed until the voltage drops to $2.75 \mathrm{~V}$. Simultaneously, for the sake of validating the correction of the developed method to the initial error, the incipient SOC of the filter-based methods are all set at $80 \%$, in contrast to the actual initial SOC of $100 \%$ with $20 \%$ error.

Fig. 7 shows the SOC estimation results based on different filers, and the statistical errors are summarized

in Table II, from which we can find that the SRCKF, AEKF and EKF can mitigate the impact of initial SOC error quickly. It respectively takes $66 \mathrm{~s}, 75 \mathrm{~s}$ and $92 \mathrm{~s}$ for the three algorithms to converge to $3 \%$ error boundary. Obviously, the SRCKF algorithm leads to the fastest convergence speed. This demonstrates that the proposed algorithm enables strong correction capability to the initial error. In addition to the autocorrection of initial error, the maximum absolute error (MAAE) of SRCKF, AEKF and EKF is $2.77 \%, 3.64 \%$ and $9.80 \%$, the mean absolute error (MEEA) is $1.22 \%, 2.19 \%$ and $2.27 \%$, and the root mean square error (RMSE) are $1.39 \%, 2.46 \%$ and $3.20 \%$, respectively. Compared with AEKF and EKF, the SRCKF raises higher estimation accuracy. The main reason can be explained as follows. For nonlinear Gaussian systems, SRCKF approximates the mean and covariance of states by the third-order spherical radial volume criterion, which can dynamically adapt to the charging and discharging characteristics of Li-ion batteries. However, the EKF and AEKF algorithms linearize the battery charging and discharging process and ignore the high-order terms, thereby discounting the filtering accuracy.

\section{SOC Evaluation at Different Ambient Temperatures}

As stated in Section II, the estimation performance of SOC is highly related to the operation temperature. 
Here, the experiments under a wide operation temperature range of $-10{ }^{\circ} \mathrm{C}$ to $60{ }^{\circ} \mathrm{C}$ are conducted to further investigate the environmental suitability of the developed estimation framework. A fully charged battery is firstly discharged at $14{ }^{\circ} \mathrm{C}$ for 1 hour, at $20^{\circ} \mathrm{C}$ for 2 hours and then at $26^{\circ} \mathrm{C}, 30{ }^{\circ} \mathrm{C}, 40{ }^{\circ} \mathrm{C}$ and $50{ }^{\circ} \mathrm{C}$ for 1 hour, respectively. The temperature evolution curve is shown in Fig. 8 (a). It can be seen that there is a fluctuation of temperature that is caused by the measure error of thermal chamber and the reaction time of temperature change. In this experiment, a hybrid cycle including UDDS and dynamic stress test (DST) is conducted. Fig. 8 (b) shows the current and voltage curves under the hybrid test cycle.

\section{1) Algorithm Verification at Low Temperature}

Fig. 9 shows the results of SOC estimation at $-10{ }^{\circ} \mathrm{C}$ and $0{ }^{\circ} \mathrm{C}$, and Table III depicts the performance evaluation results, in which the "Reference" represents the SOC value obtained by the Coulomb counting method at corresponding temperature, "T-SRCKF" means the result of SOC estimation obtained by the proposed SRCKF algorithm with the temperature compensation ECM, "SRCKF" refers to the SOC recognized by the SRCKF without temperature compensation, and the model parameters are identified at $25^{\circ} \mathrm{C}$. It can be obviously found from Fig. 9 that the estimated value based on the presented framework and the traditional SRCFK can trace the reference SOC smoothly. The estimation error of the presented framework is less than $1 \%$ at $-10{ }^{\circ} \mathrm{C}$ and $0{ }^{\circ} \mathrm{C}$ within the full SOC operating range; and in contrast, the MAAE of SRCKF method without temperature compensation are respectively $3.48 \%$ and $3.49 \%$ at $-10{ }^{\circ} \mathrm{C}$ and $0{ }^{\circ} \mathrm{C}$, demonstrating the necessity of adjusting the model parameters according to the ambient temperature for improving SOC estimation accuracy.

\section{2) Algorithm Verification at High Temperature}

The results of SOC estimation at $50{ }^{\circ} \mathrm{C}$ and $60{ }^{\circ} \mathrm{C}$ are shown in Fig. 10, and the detailed performance is exhibited in Table III. The results indicate that although the battery works at a high temperature (more than 50 $\left.{ }^{\circ} \mathrm{C}\right)$, the T-SRCKF algorithm can still estimate the SOC accurately. Concretely, the MAAE, MEAE and RMSE at $50{ }^{\circ} \mathrm{C}$ are $0.56 \%, 0.34 \%$ and $0.38 \%$, respectively; and meanwhile, the MAAE, MEAE and RMSE at $60{ }^{\circ} \mathrm{C}$ are $0.32 \%, 0.21 \%$ and $0.23 \%$, obviously lower than those of traditional SRCKF method. According to the estimation results at low and high temperatures, it is noticeable that large error appears at lower temperature due to serious polarization in this case. Additionally, lower range of SOC leads to higher SOC error. However, no matter what temperature the battery works at, the designed T-SRCKF algorithm can effectively improve the estimation performance in full SOC range.

\section{3) Algorithm Verification at Time-Varying Temperature Conditions}


For EVs, the working environment temperature is not constant. Meanwhile, the battery temperature will continuously increase with the EV operation until the thermal balance is reached. To further validate the effectiveness and adaptability of T-SRCKF algorithm, the cycle test under dynamic temperature conductions is conducted. Fig. 8 shows the experimental temperature and current variation curves. Note that the reference SOC is calculated via the ampere hour integration method, and the maximum discharge capacity constantly changes and updates with the variation of ambient temperature. Fig. 11 and Table III summarize the SOC prediction results, which show that the estimation error is less than 1.8\%, and the MAAE, MEAE and RMSE are 1.75\%, $0.83 \%$ and $1.03 \%$, respectively, slightly higher than that of constant temperature condition. This is mainly because the time-varying temperature can lead to large fluctuation of internal model parameters. The SRCKF algorithm without temperature compensation can also follow the variation of reference value; however, it incurs large error and fluctuation, and the MAAE is close to $3 \%$. The experimental validations authenticate the proposed framework can better adapt to the temperature variation in practical application and precisely predict the SOC under complex temperature conditions.

\section{CONCLUSION}

In this study, an advanced framework based on square root cubature Kalman filter is proposed to enhance the estimation accuracy of battery state of charge in complex temperature environment. In the proposed framework, an electric model integrating temperature variation in a wide range of $-20^{\circ} \mathrm{C}$ to $60{ }^{\circ} \mathrm{C}$ is constructed to simulate its electric characteristics. The square root cubature Kalman filter algorithm is then exploited to estimate the state of charge considering the accuracy and computational efficiency. The precision and error correction ability of the devised framework are compared with traditional filter-based methods at $25^{\circ} \mathrm{C}$, and the reference state of charge trajectory can be precisely followed in the whole operation range by the proposed method. In addition, more detail experiments are conducted at different working conditions, and the results elucidate that the developed framework can maintain the maximum absolute error, mean absolute error and root mean square error within $1.75 \%, 0.83 \%$, and $1.03 \%$, respectively, even with time-varying temperatures from $10{ }^{\circ} \mathrm{C}$ to $50{ }^{\circ} \mathrm{C}$, verifying the preferable estimation capability and robust environmental adaptation of the proposed method. To conclude, the validation results indicate that the presented framework can estimate the state of charge with high accuracy under wide dynamic temperature conditions.

Although the present estimation framework can effectively adapt to the temperature, the capacity 
degradation will show an important impact on the SOC estimation accuracy. Hence, a joint estimation method of capacity and state of charge will be certainly investigated in our future work. Moreover, the state of charge estimation of battery pack on the account of single cell estimation also needs to be tackled in our next step research.

\section{ACKNOWLEDGEMENTS}

This work is supported in part by the National Science Foundation under Grant 61763021, in part by the National Key R\&D Program of China under Grant 2018YFB0104000 and Grant 2018YFB0104500, and in part by in part by the EU-funded Marie Skłodowska-Curie Individual Fellowships Project under Grant 845102 HOEMEV-H2020-MSCA-IF-2018.

\section{DATA ACCESSIBILITY}

The data that support the findings of this study are available from the corresponding authors upon reasonable request.

\section{References}

[1] X. Zhang, Y.J. Wang, D. Yang, and Z.H. Chen, "An on-line estimation of battery pack parameters and state-ofcharge using dual filters based on pack model," Energy, vol. 115, pp. 219-229, 2016.

[2] X. Shu, G. Li, J. Shen, Z. Lei, Z. Chen, and Y. Liu, "A uniform estimation framework for state of health of lithiumion batteries considering feature extraction and parameters optimization," Energy, vol. 204, pp. 0360-5442, 2020.

[3] M. Shen and Q. Gao, "A review on battery management system from the modeling efforts to its multiapplication and integration," International Journal of Energy Research, vol. 43, pp. 5042-5075, 2019.

[4] X.S. Hu, F. Feng, K.L. Liu, L. Zhang, J.L. Xie, and B. Liu, "State estimation for advanced battery management: Key challenges and future trends," Renewable \& Sustainable Energy Reviews, vol. 114, pp. 1364-0321, 2019.

[5] X. Tang, C. Zou, T. Wik, K. Yao, and F. Gao, "Run-to-run control for active balancing of lithium iron phosphate battery packs," IEEE Transactions on Power Electronics, vol. 35, pp. 1499-1512, 2019.

[6] Z. Chen, H.J. Hu, Y.T. Wu, R.X. Xiao, J.W. Shen, and Y.G. Liu, "Energy Management for a Power-Split Plug-In Hybrid Electric Vehicle Based on Reinforcement Learning," Applied Sciences-Basel, vol. 8, pp. 2076-3417, 2018.

[7] X. Shu, G. Li, J. Shen, Z. Lei, Z. Chen, and Y. Liu, "An adaptive multi-state estimation algorithm for lithium-ion batteries incorporating temperature compensation," Energy, vol. 207, pp. 0360-5442, 2020.

[8] J. Chen, Q. Ouyang, C.F. Xu, and H.Y. Su, "Neural Network-Based State of Charge Observer Design for LithiumIon Batteries," Ieee Transactions on Control Systems Technology, vol. 26, pp. 313-320, 2018.

[9] Y. Wang, J. Tian, Z. Sun, L. Wang, R. Xu, M. Li, and Z. Chen, "A comprehensive review of battery modeling and state estimation approaches for advanced battery management systems," Renewable \& Sustainable Energy Reviews, vol. 131, pp. 1364-0321, 2020.

[10] X. Ding, and D. Zhang, "A novel active equalization topology for series-connected lithium-ion battery packs," 2018 IEEE Energy Conversion Congress and Exposition (ECCE), IEEE, 2018.

[11] Z.B. Wei, C.F. Zou, F. Leng, B.H. Soong, and K.J. Tseng, "Online Model Identification and State-of-Charge Estimate for Lithium-Ion Battery With a Recursive Total Least Squares-Based Observer," Ieee Transactions on Industrial Electronics, vol. 65, pp. 1336-1346, 2018.

[12] X. Shu, G. Li, J. Shen, W. Yan, Z. Chen, and Y. Liu, "An adaptive fusion estimation algorithm for state of charge 
of lithium-ion batteries considering wide operating temperature and degradation," Journal of Power Sources, vol. 462, pp. 0378-7753, 2020.

[13] R. Xiong, J.Y. Cao, Q.Q. Yu, H.W. He, and F.C. Sun, "Critical Review on the Battery State of Charge Estimation Methods for Electric Vehicles," Ieee Access, vol. 6, pp. 1832-1843, 2018.

[14] X. Tang, Y. Wang, C. Zou, K. Yao, Y. Xia, and F. Gao, "A novel framework for Lithium-ion battery modeling considering uncertainties of temperature and aging," Energy Conversion and Management, vol. 180, pp. 162-170, 2018.

[15] X. Tang, F. Gao, C. Zou, K. Yao, W. Hu, and T. Wik, "Load-responsive model switching estimation for state of charge of lithium-ion batteries," Applied Energy, vol. 238, pp. 423-434, 2019.

[16] C. Huang, Z.H. Wang, Z.H. Zhao, L. Wang, C.S. Lai, and D. Wang, "Robustness Evaluation of Extended and Unscented Kalman Filter for Battery State of Charge Estimation," Ieee Access, vol. 6, pp. 27617-27628, 2018.

[17] B. Ning, B. Cao, B. Wang, and Z. Zou, "Adaptive sliding mode observers for lithium-ion battery state estimation based on parameters identified online," Energy, vol. 153, pp. 732-742, 2018.

[18] B. Duan, Q. Zhang, F. Geng, and C.H. Zhang, "Remaining useful life prediction of lithium-ion battery based on extended Kalman particle filter," International Journal of Energy Research, vol. 44, pp. 1724-1734, 2020.

[19] W.Y. Kim, P.Y. Lee, J. Kim, and K.S. Kim, "A nonlinear-model-based observer for a state-of-charge estimation of a lithium-ion battery in electric vehicles," Energies, vol. 12, pp. 1996-1073, 2019.

[20] H.W. He, H.Z. Qin, X.K. Sun, and Y.P. Shui, "Comparison Study on the Battery SoC Estimation with EKF and UKF Algorithms," Energies, vol. 6, pp. 5088-5100, 2013.

[21] D. Xile, Z. Caiping, and J. Jiuchun, "Evaluation of SOC Estimation Method Based on EKF/AEKF under Noise Interference," Energy Procedia, vol. 152, pp. 520-525, 2018.

[22] I. Arasaratnam and S. Haykin, "Cubature Kalman Filters," Ieee Transactions on Automatic Control, vol. 54, pp. 1254-1269, 2009.

[23] J. Peng, J. Luo, H. He, and B. Lu, "An improved state of charge estimation method based on cubature Kalman filter for lithium-ion batteries," Applied Energy, vol. 253, pp. 0306-2619, 2019.

[24] L.Q. Zhao, J.L. Wang, T. Yu, H. Jian, and T.J. Liu, "Design of adaptive robust square-root cubature Kalman filter with noise statistic estimator," Applied Mathematics and Computation, vol. 256, pp. 352-367, 2015.

[25] Y.T. Huo, W. Hu, Z. Li, and Z.H. Rao, "Research on parameter identification and state of charge estimation of improved equivalent circuit model of Li-ion battery based on temperature effects for battery thermal management," International Journal of Energy Research, pp. 1099-114X, 2020.

[26] K.T. Lee, M.J. Dai, and C.C. Chuang, "Temperature-Compensated Model for Lithium-Ion Polymer Batteries With Extended Kalman Filter State-of-Charge Estimation for an Implantable Charger," Ieee Transactions on Industrial Electronics, vol. 65, pp. 589-596, 2018.

[27] Z.X. Liu, Z. Li, J.B. Zhang, L.S. Su, and H. Ge, "Accurate and Efficient Estimation of Lithium-Ion Battery State of Charge with Alternate Adaptive Extended Kalman Filter and Ampere-Hour Counting Methods," Energies, vol. 12, pp. 1996-1073, 2019.

[28] X.G. Wu, X.F. Li, and J.Y. Du, "State of Charge Estimation of Lithium-Ion Batteries Over Wide Temperature Range Using Unscented Kalman Filter," Ieee Access, vol. 6, pp. 41993-42003, 2018.

[29] Z. Chen, X. Shu, R.X. Xiao, W.S. Yan, Y.G. Liu, and J.W. Shen, "Optimal charging strategy design for lithiumion batteries considering minimization of temperature rise and energy loss," International Journal of Energy Research, vol. 43, pp. 4344-4358, 2019.

[30] H. Pang, L. Guo, L.X. Wu, and X.F. Jin, "An enhanced temperature-dependent model and state-of-charge estimation for a Li-on battery using extended Kalman filter," International Journal of Energy Research, vol. 44, pp. 7254-7267, 2020.

[31] F. Guo, G. Hu, P. Zhou, J. Hu, and Y. Sai, "State of charge estimation in electric vehicles at various ambient temperatures," International Journal of Energy Research, vol. 44, pp. 7357-7370, 2020.

[32] H.F. Dai, B. Jiang, and X.Z. Wei, "Impedance Characterization and Modeling of Lithium-Ion Batteries 
Considering the Internal Temperature Gradient," Energies, vol. 11, pp. 1996-1073, 2017.

[33] Z.B. Wei, J.Y. Zhao, C.F. Zou, T.M. Lim, and K.J. Tseng, "Comparative study of methods for integrated model identification and state of charge estimation of lithium-ion battery," Journal of Power Sources, vol. 402, pp. 189. 197, 2018.

[34] Y. Luo, P.W. Qi, Y.Z. Kan, J.Y. Huang, H. Huang, J.W. Luo, J.N. Wang, Y.H. Wei, R.J. Xiao, and S. Zhao, "State of charge estimation method based on the extended Kalman filter algorithm with consideration of time-varying battery parameters," International Journal of Energy Research, pp. 1099-114X, 2020.

[35] Z. Chen, X. Shu, X.Y. Li, R.X. Xiao, and J.W. Shen, "LiFePO4 battery charging strategy design considering temperature rise minimization," Journal of Renewable and Sustainable Energy, vol. 9, pp. 1941-7012, 2017.

[36] C. Zhang, K. Li, L. Pei, and C.B. Zhu, "An integrated approach for real-time model-based state-of-charge estimation of lithium-ion batteries," Journal of Power Sources, vol. 283, pp. 24-36, 2015.

[37] M.D. Brondani, A. Sausen, P.S. Sausen, and M.O. Binelo, "Parameter estimation of lithium ion polymer battery mathematical model using genetic algorithm," Computational \& Applied Mathematics, vol. 37, pp. 296-313, 2018. 
Table I. Specification of test batteries.

\begin{tabular}{lc}
\hline Specifications & Value \\
\hline Nominal capacity & $4 \mathrm{Ah}$ \\
Voltage range & 2.75 to $4.2 \mathrm{~V}$ \\
Allowed charge temperature & 0 to $45^{\circ} \mathrm{C}$ \\
Allowed discharge temperature & -20 to $60^{\circ} \mathrm{C}$ \\
\hline
\end{tabular}

Table II. Statistical errors with different filter-based methods.

\begin{tabular}{ccccc}
\hline Algorithm & $\begin{array}{c}\text { Convergence } \\
\text { time }(\mathrm{s})\end{array}$ & MAAE $(\%)$ & MEAE (\%) & RMSE (\%) \\
\hline EKF & 92 & 9.80 & 2.27 & 3.20 \\
AEKF & 75 & 3.64 & 2.19 & 2.46 \\
SRCKF & 66 & 2.77 & 1.22 & 1.39 \\
\hline
\end{tabular}

Table III. Statistical errors at different temperatures.

\begin{tabular}{cccc}
\hline Temperature $\left({ }^{\circ} \mathrm{C}\right)$ & MAAE $(\%)$ & MEAE $(\%)$ & RMSE $(\%)$ \\
\hline-10 & 0.59 & 0.35 & 0.42 \\
0 & 0.68 & 0.44 & 0.51 \\
50 & 0.65 & 0.37 & 0.47 \\
60 & 0.32 & 0.21 & 0.27 \\
Time-Varying & 1.75 & 0.83 & 1.03 \\
\hline
\end{tabular}

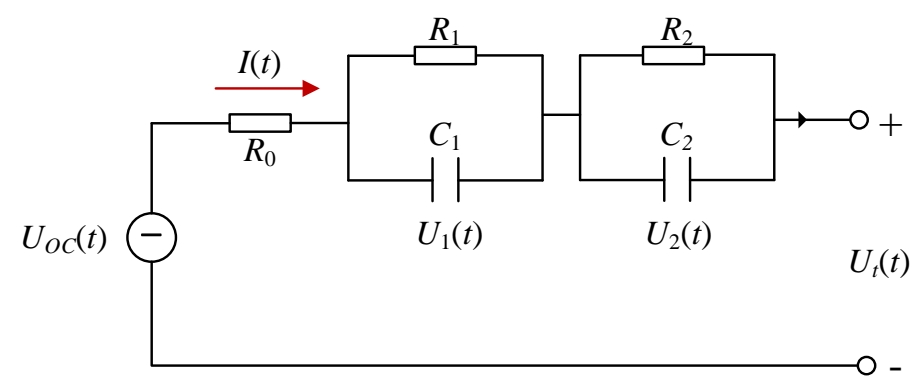

Fig. 1. Illustration of two-order RC ECM.
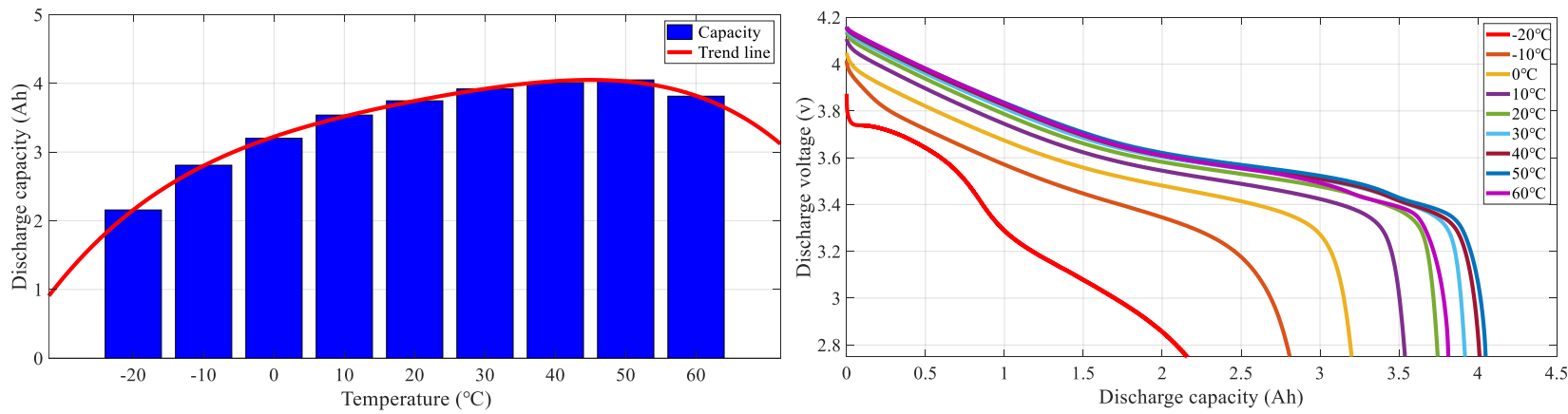

Fig. 2. Discharge capacity at different temperatures. (a) Discharge capacity. (b) Discharge voltage curve.

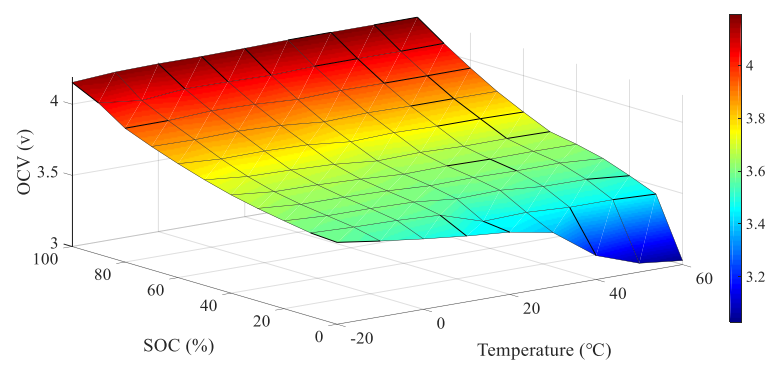

Fig. 3. The three-dimensional response surface between OCV, SOC and temperature. 


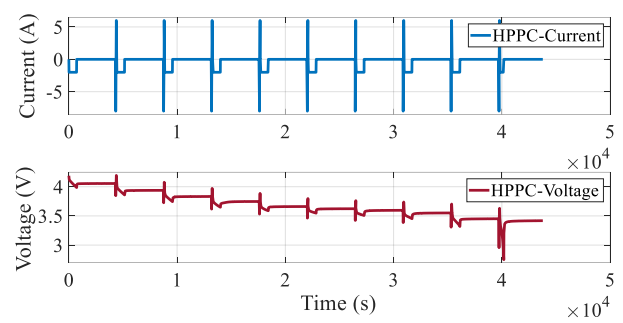

(a)

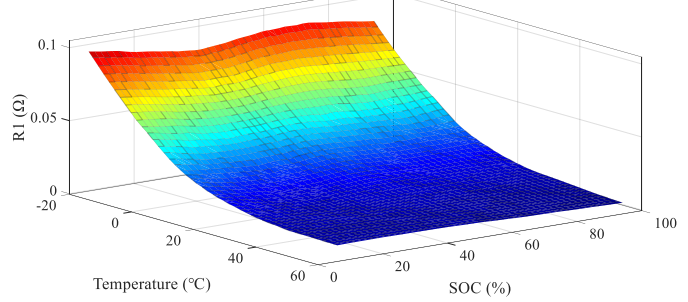

(c)

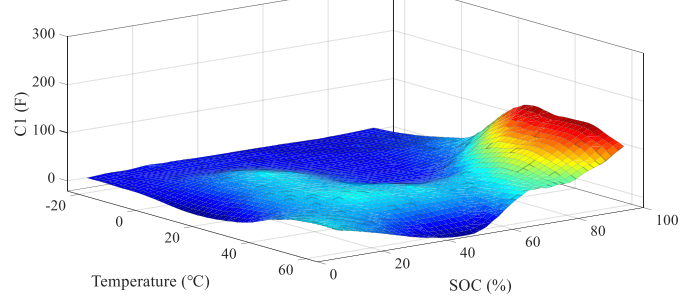

(e)

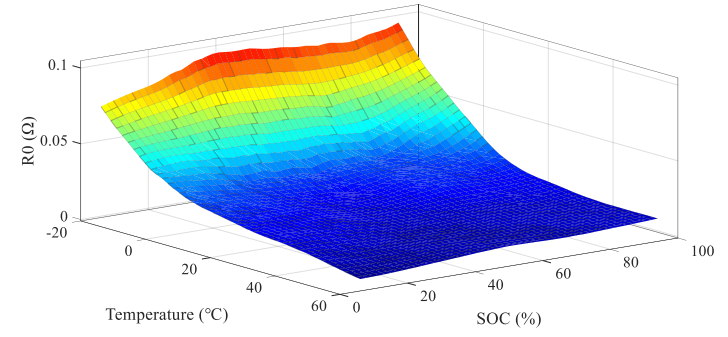

(b)

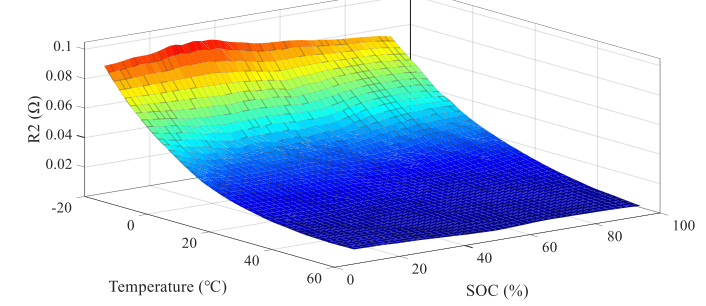

(d)

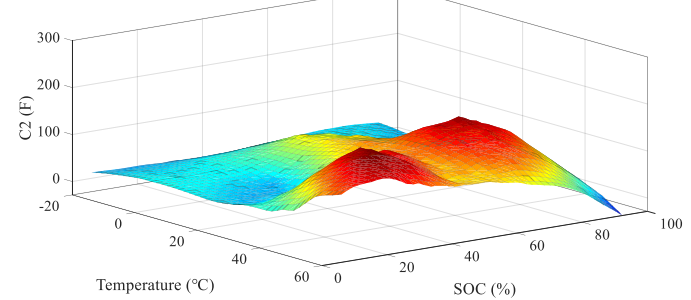

(f)

Fig. 4. The identification results of model parameters. (a) HPPC result at $25^{\circ} \mathrm{C}$. (b) $\mathrm{R}_{0}-\mathrm{SOC}-\mathrm{T}$. (c) $\mathrm{R}_{1}$-SOC-T. (d) $\mathrm{R}_{2}$-SOC-T. (e) $\mathrm{C}_{1}$-SOC-T. (f) $\mathrm{C}_{2}$-SOC-T.

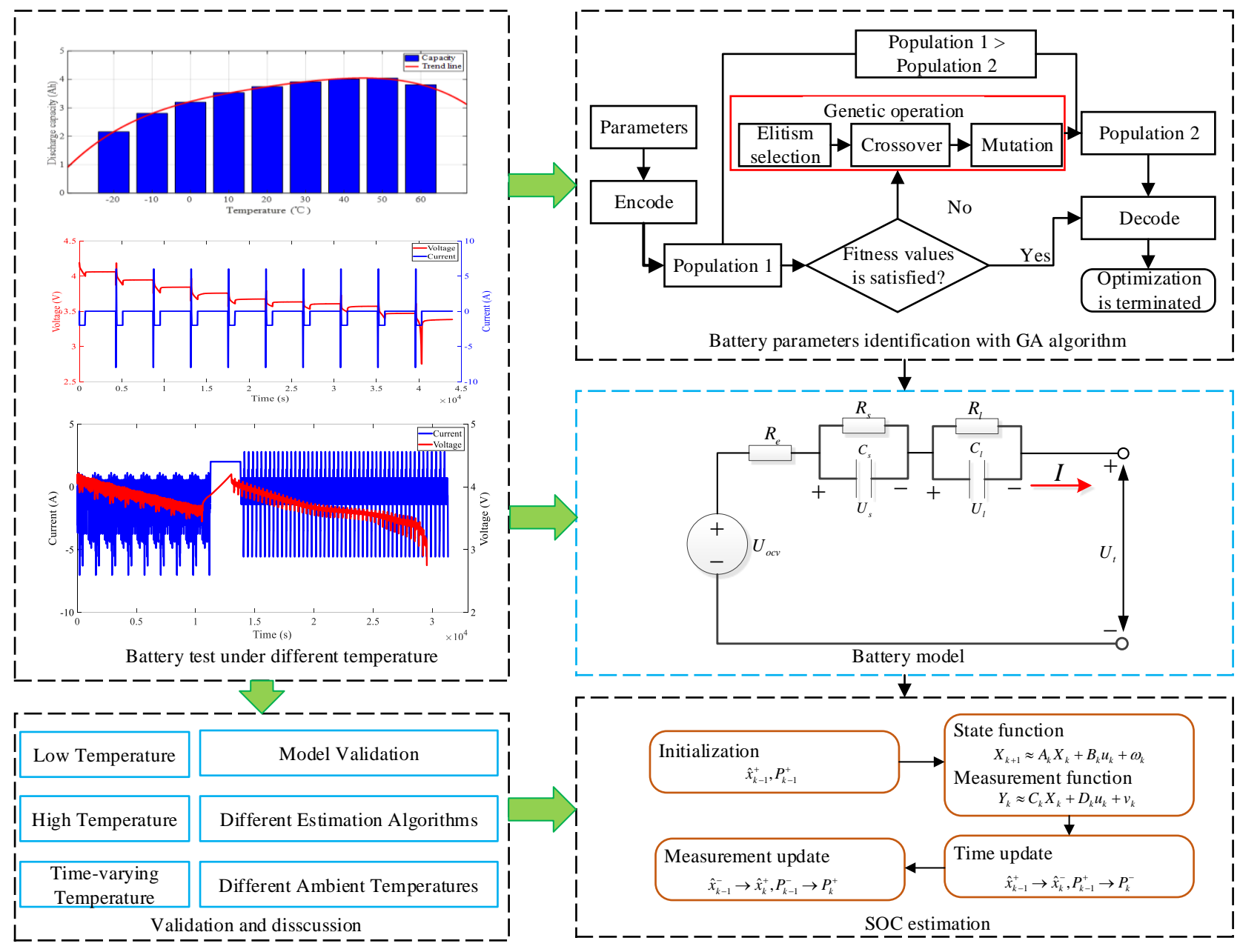

Fig. 5. The flowchart of SOC estimation framework. 


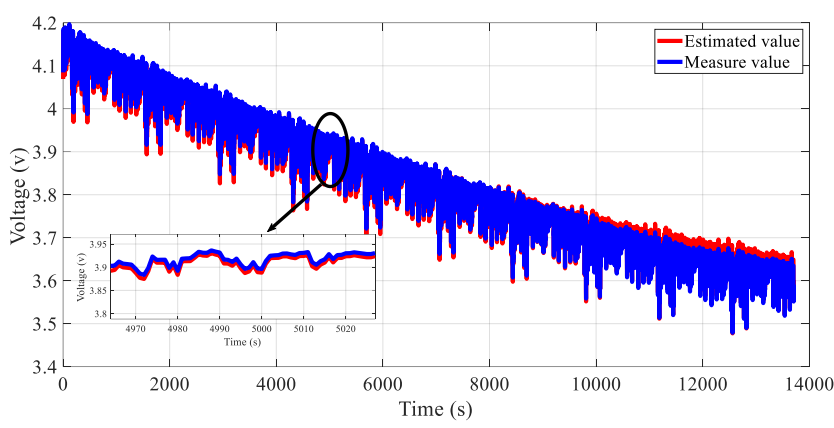

(a)

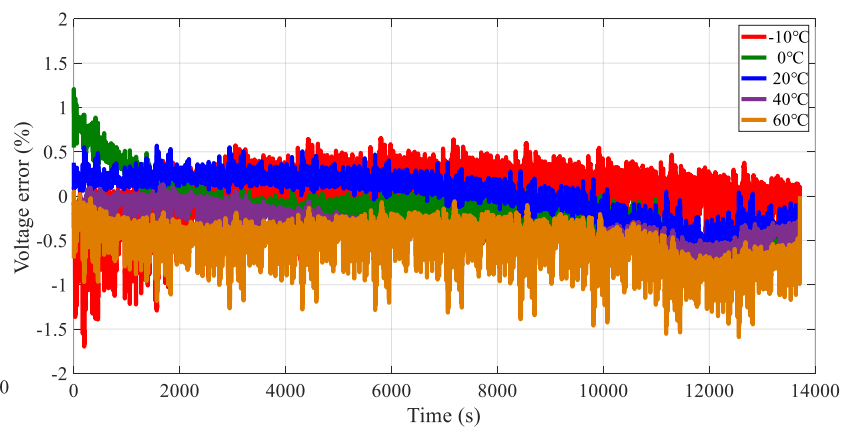

(b)

Fig. 6. The model verification results at different temperatures. (a) Voltage estimation results at $20{ }^{\circ} \mathrm{C}$. (b) Voltage estimation error at different temperatures.

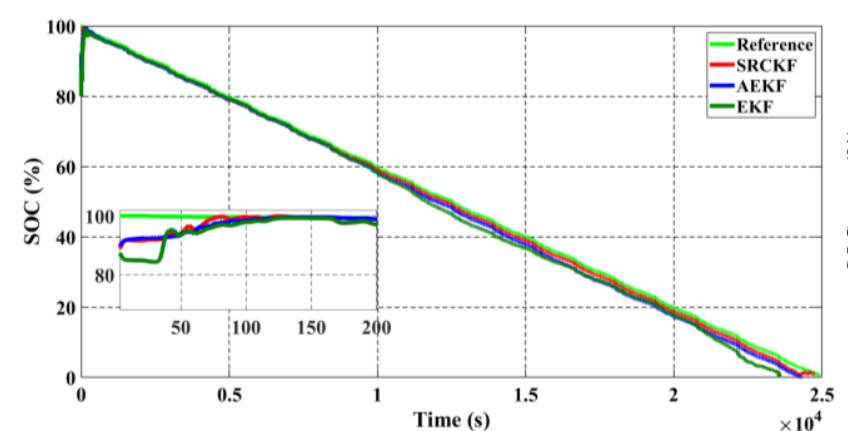

(a)

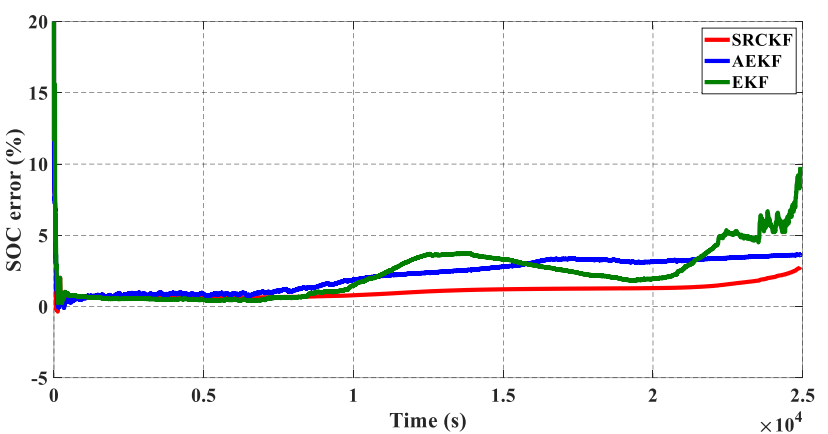

(b)

Fig. 7. The SOC estimation results with different filter-based algorithms. (a) Estimation results. (b) Estimation error.

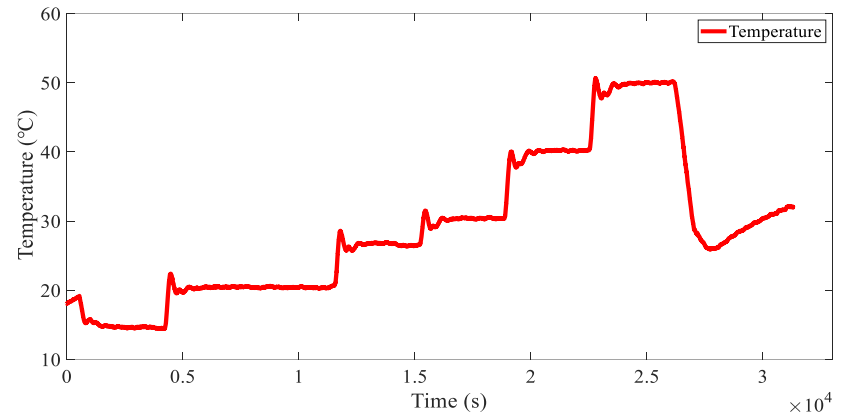

(a)

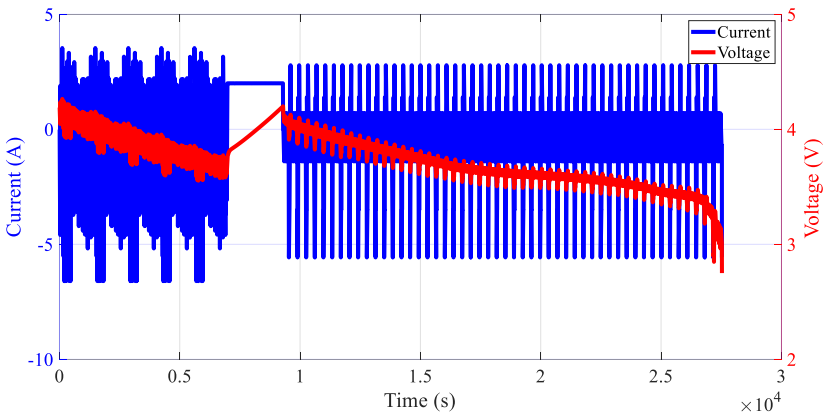

(b)

Fig. 8. UDDS-DST test cycle at time-varying temperature. (a) Time-varying temperature curve. (b) The current and voltage curve of battery.

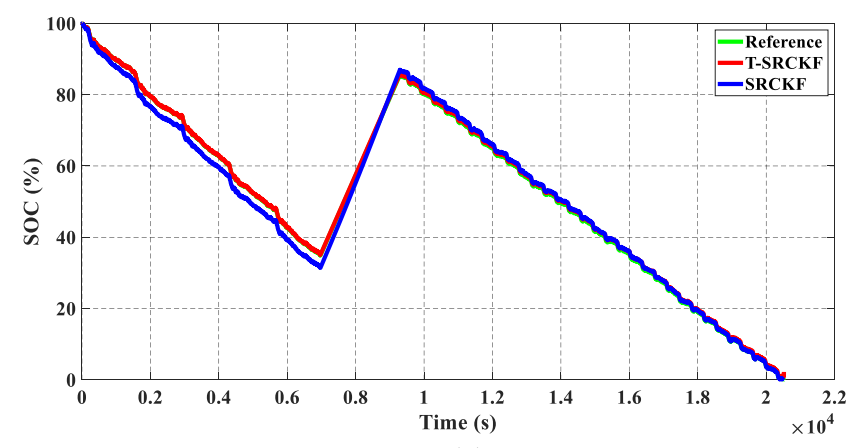

(a)

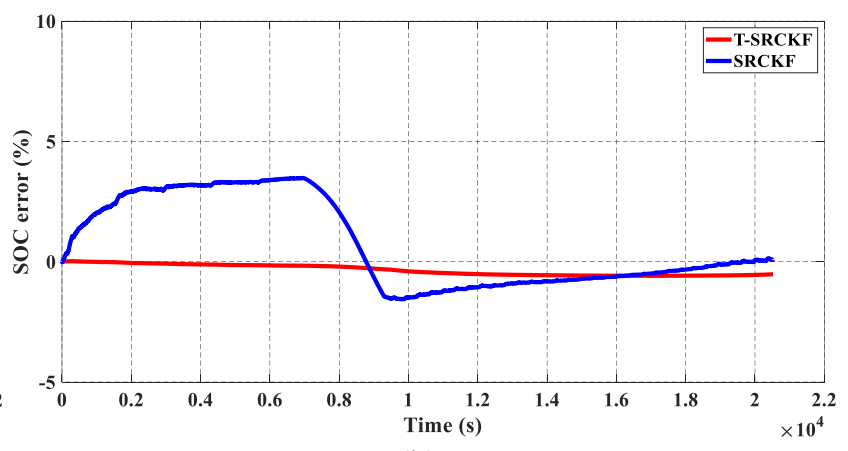

(b) 


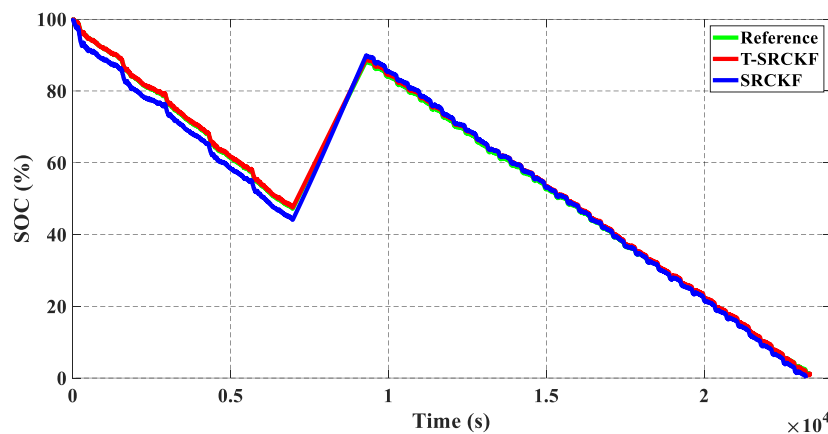

(c)

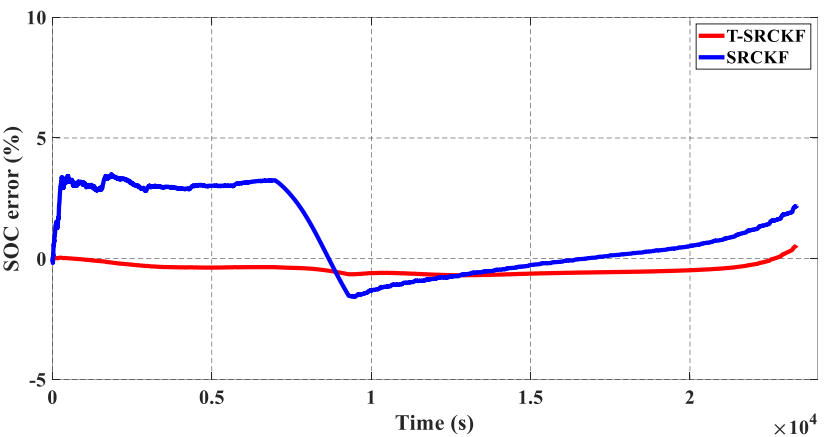

(d)

Fig. 9. SOC estimation results at low temperature environment. (a) Estimation results at $-10{ }^{\circ} \mathrm{C}$. (b) Estimation error at $-10{ }^{\circ} \mathrm{C}$. (c) Estimation results at $0{ }^{\circ} \mathrm{C}$. (d) Estimation error at $0{ }^{\circ} \mathrm{C}$.

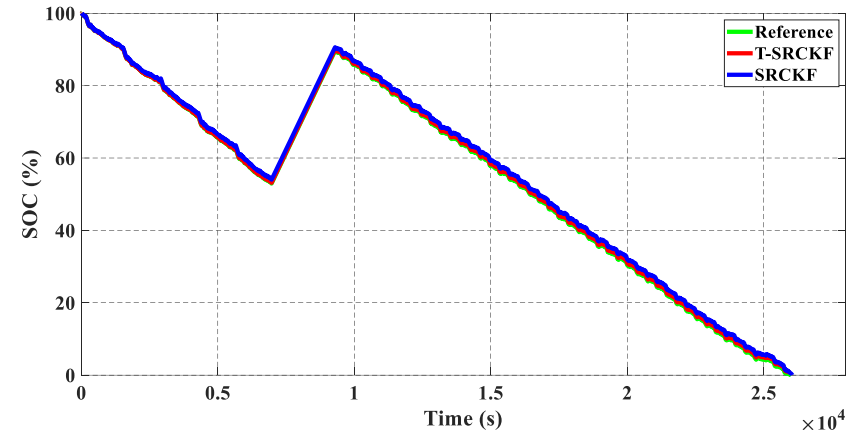

(a)

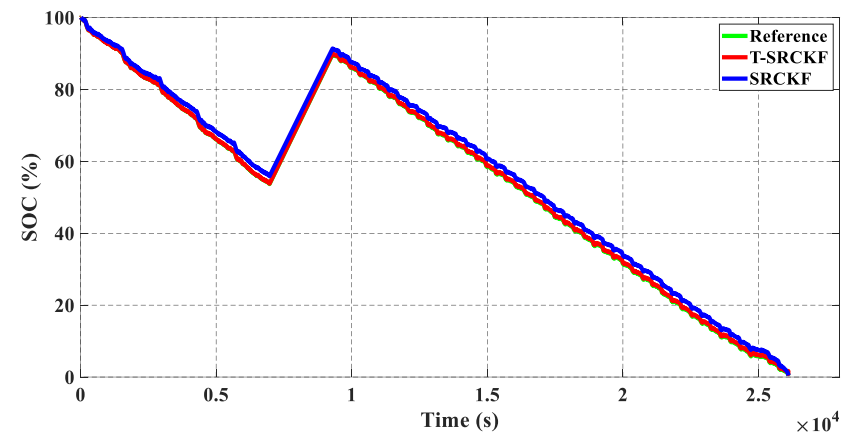

(c)

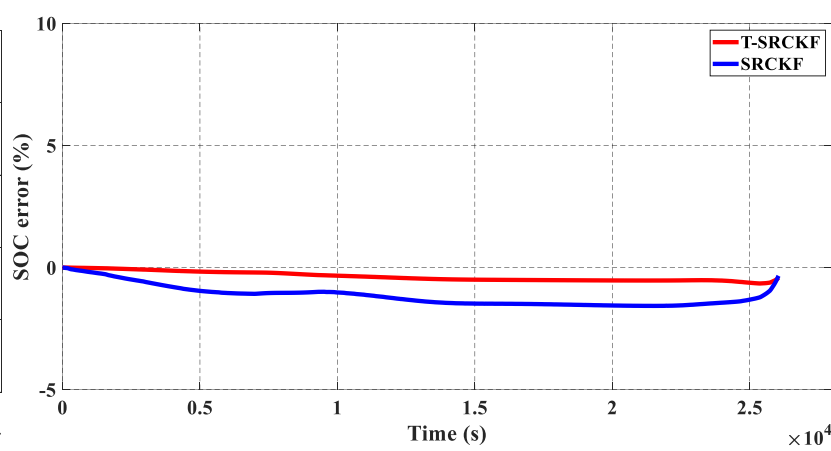

(b)

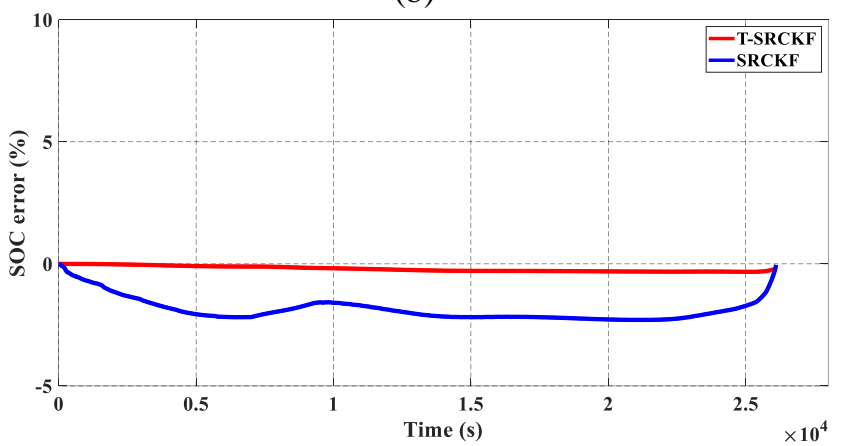

(d)

Fig. 10. The SOC estimation results at high temperature environment. (a) Estimation results at $50{ }^{\circ} \mathrm{C}$. (b) Estimation error at $50{ }^{\circ} \mathrm{C}$. (c) Estimation results at $60{ }^{\circ} \mathrm{C}$. (d) Estimation error at $60{ }^{\circ} \mathrm{C}$.

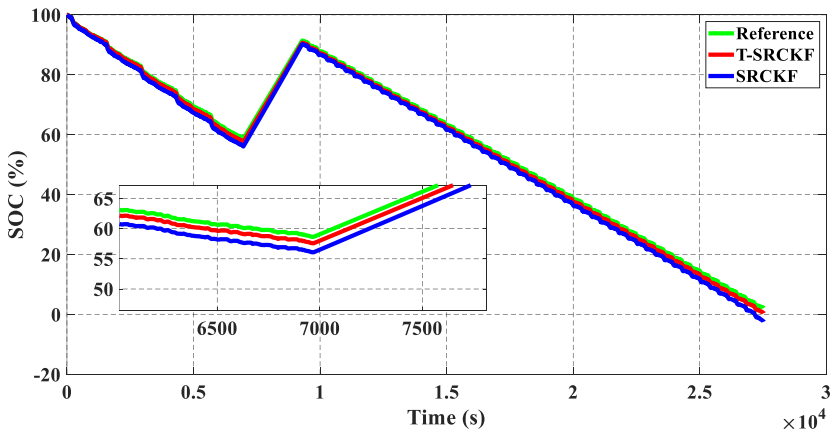

(a)

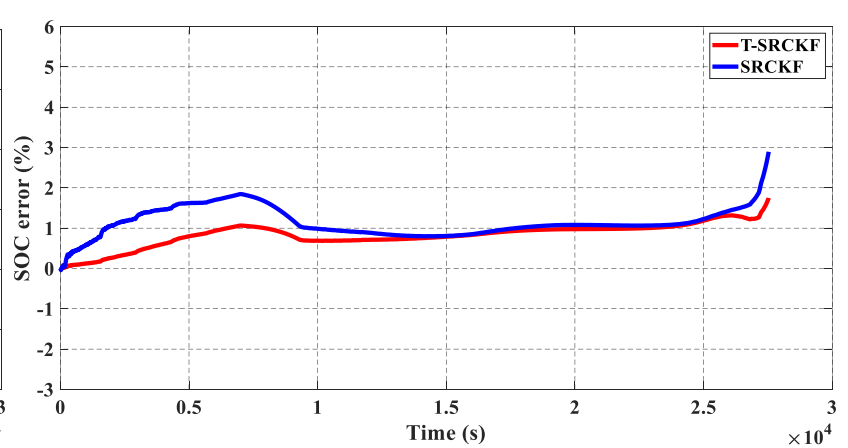

(b)

Fig. 11. SOC estimation results under time-varying temperature. (a) Estimation results. (b) Estimation error. 